\title{
AUDIO CAPTION GENERATION FROM IMAGES USING DEEP LEARNING
}

\author{
${ }^{1}$ Omprakash Yadav, ${ }^{2}$ Atharva Jadhav, ${ }^{2}$ Abdul Hannan Sunsara, ${ }^{2}$ Idris Vohra \\ ${ }^{1}$ Professor, ${ }^{2}$ Students, Department of Computer Engineering, Xavier Institute of Engineering, Mumbai, India \\ 1omprakash.y@xavier.ac.in, ${ }^{2}$ atharvasj780@gmail.com, ${ }^{3}$ abdul.hannan13300@gmail.com, ${ }^{4}$ idrees786.iv@gmail.com
}

\begin{abstract}
Visually impaired individuals face various types of difficulties as they cannot visualize the natural environment. To overcome this problem, the proposed system would automatically generate captions for an input images and convert the generated caption to an audio format so that visually impaired individuals can listen to the generated captions. Captioning is performed using Deep Learning algorithm Convolution Neural Network (CNN), Recurrent Neural Network (RNN) and Long Short-Term Memory.
\end{abstract}

Keywords: Deep Learning, RNN, LSTM, CNN.

\section{INTRODUCTION}

Vision deficiency, also known as loss of vision or vision impairment, is nothing but a vision disability which has no immediate remedies of any kind. It is something that any person does not wish to have. Millions of individuals are visually impaired globally, according to the World Health Organization(WHO).Without one of the most useful sensory organs in a technologically developed environment where even the smallest piece of work needs hearing, it is very hard to survive. In today's world where the technology sector is growing, many developments can be made where the technology can provide aid to the visually impaired people. One technique is to detect items in an image and have a meaningful caption that will be in the form of audio that would enable visually disabled people to associate all object to an image. Generating an image caption requires a number of tasks, such as understanding the higher level of semantics, and then explaining the semantics in a sentence that humans can understand. Communication in human beings takes place with the aid of natural language, so it becomes a challenge to create a system that creates explanations that human beings can understand. The goal of this paper is to detect, recognize and generate images using Deep Learning algorithms.

\section{LITERATURE REVIEW}

The extraction of natural language descriptions from a photo is a fairly new concept, although a lot of work has been done in this area. This segment will give you a brief understanding of the work that has been done to date.

In this [1], the system generates captions automatically for the news articles. The caption is generated from the data set of various news articles

along with an image embedded in them. This model consists of two phases of collection of material and surface realization. Selection of content defines that the image and the corresponding article are about, while surface knowledge determines how to convey the chosen content.

This paper [2], presents a model that generates captions automatically for news articles with an embedded image in it. Captions for the news articles are generated by using the stemming algorithm and frequency ranking calculation.

This[3] framework is designed to help mobile users create captions for their images. Users must upload a photo to a cloud service where a range of parallel modules, such as face detection, GPS, date-time, scene recognition , are used to identify a variety of individuals and relationships. Module outputs are combined to create a large collection of captions.

Deep Visual-Semantic Alignments for Generating Image Descriptions model can automatically generate captions for given images using multimodal RNN [4]. This model aligns sentence snippets to the visual regions that they describe through multimodal embedding then uses this as an input to multimodal RNN that will generate snippets.

In this [5] presented model is based on the concept 'Show and Tell'. This model had used CNN and LSTM to generate a caption. This model has been implemented by using various datasets to obtain more accurate results.

In the proposed model [6], which takes input as an audio file and converts it into text. The conversion is done by using an encoder-decoder scheme with an attentional layer in between them. The method was evaluated using a commercial dataset of recordings, each of which is associated with a textual description (caption) within the dataset.

An automatic caption generation model has been 
implemented by using Recurrent Neural Network and LSTM with additional Read-Only units [7]. This model is trained with the MSCOCO dataset which generates more accurate captions by using all these technologies together.

\section{METHODOLOGY USED}

The photo caption generation problem can be addressed by using a standard encoder-decoder RNN architecture which involves two elements which are the encoder and the decoder. The encoder reads the input image and produces a fixed-length vector using an internal representation, and the decoder takes the encoded input and generates a text summary.

In short, the generated output is expected to describe in a single sentence what is shown in the image such as the objects present, the properties, actions being performed and interaction between objects, etc. A pre-trained convolutional neural network (CNN) model can be used to encode the images and RNN, such as a Long ShortTerm Memory network can be used to encode the generated text sequence or generate the next word in the sequence. Mentioned below are the technical approaches in detail which are used to build a model.

\section{A. CONVOLUTIONNEURAL NETWORK}

A Convolution Neural Network is a deep learning algorithm that can take input in the form of the image, assign the learnable weights and biases to various objects in an image and then make one object different from another A much lower pre-processing is required compared to other classification algorithms. The architecture of this network is similar to that of the pattern of neuron communication in the human brain. Individually, neurons can respond to stimulus only in the restricted area of the visual field known as the Receptive Field. The array of such fields is then overlapped to fill the entire visual region. A collection of such fields then overlaps to cover the entire visual area. Also, there is an "observation window" from which the model tries to learn the weights of the filter. The weights are shared for each convolution. It ensures translational equivariance by allowing the model to recognize objects wherever they show up in the image.

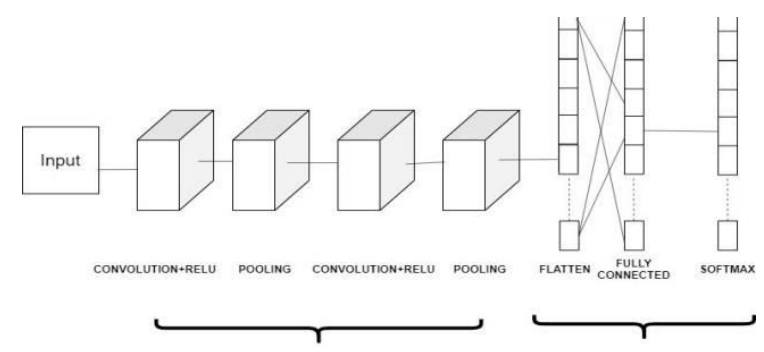

Fig. 1 Sequence of transformations involved in the convolutional neural network [11].

\section{B. RECURRENT NEURAL NETWORK}

The recurrent neural network architecture advances in being able to capture information about past states to inform current predictions via its memory cell status [1]. Generally, in traditional neural networks, all the inputs and outputs are not dependent of each other. The problem with feed-forward neural networks is that they need a fixed-sized input and give a fixed-sized output. They do not capture sequences or information about time series nor do the account for memory. But in some cases, like when it is required to predict the next word of a sentence, the previous words are required and therefore there is a need to remember the previous words. Thus, RNN was introduced which solved the issue. It can take variable-sized input and give variable-sized output and it works well with time series data as well. One of the most important features of RNN is the hidden state which remembers some information about a sequence. RNN has a 'memory' because of which it remembers all the information about what has been calculated. For each input, it uses the same parameters as it performs the same task on all the inputs or hidden layers to produce the output. And doing this, it reduces the complexity of parameters.

The intuitive representation of RNN is shown in Fig.

2. The basic formula of RNN is the recursive formula which is as follows:

$\mathrm{St}=\mathrm{F}_{\mathrm{w}}\left(\mathrm{S}_{\mathrm{t}}-1, \mathrm{X}_{\mathrm{t}}\right)$

where $\mathrm{St}$ is the new state at time $\mathrm{t}, \mathrm{F}_{\mathrm{w}}$ is the recursive function, St is the state at time $t-1$ and $X_{t}$ is the input at time $\mathrm{t}$.

To deal with the vanishing gradient problem faced by RNN, Long Short-Term Memory (LSTM) was

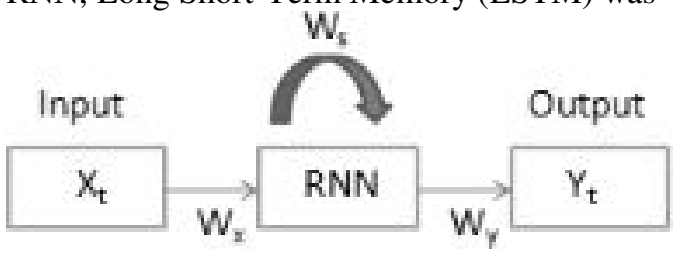

Fig. 2 Representation of RNN

introduced. Suppose if there is a deeper network with one input layer, more than one hidden layer and one output layer then RNN basically performs the following actions:

1. Firstly, the conversion of independent activation into dependent activation takes place by providing the same weights and biases to all the layers. It would help to reduce the difficulty of increasing parameters and memorize each previous output by giving each output as input to the next hidden layer.

2.Therefore all the hidden layers can be joined together such that the weights and biases of all the hidden layers are the same into a single recurrent layer. Fig. 3 shows a simplified version of RNN model. 


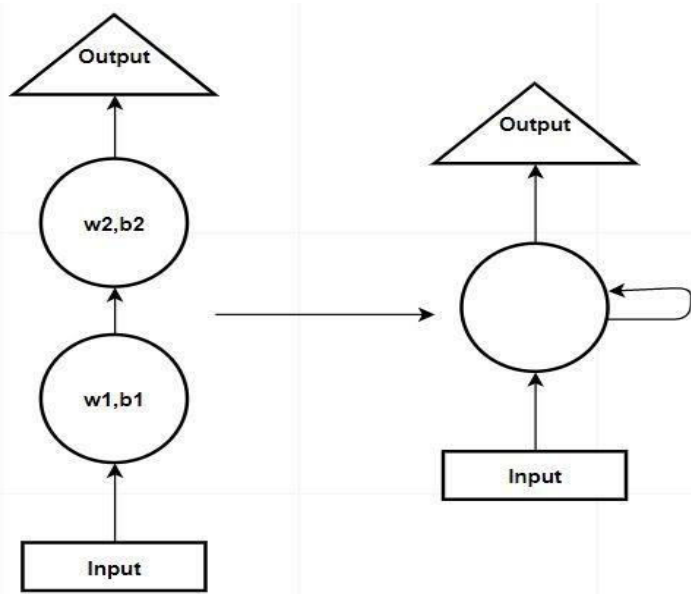

Fig. 3 Simplified form of RNN model

\section{DATASET}

We have used the Flicker $8 \mathrm{k}$ dataset to train our model. This dataset contains two types of data, image, and caption(text). This dataset contains 8092 images in JPEG format with different shapes and sizes. Out of which 6000 images will be used for training, 1000 for tests and the last 1000 for development. Fliker8k dataset also contains text data. In that dataset, Fliker8k.token.txt file contains 5 captions for each image i.e., 40460 captions.

\section{TEXT TO SPEECH CONVERSION}

To convert generated captions into audio we have used Android Text To Speech class. Android allows us not only to convert the text into audio but also, we can convert it into different languages. In order to use this class, we need to first initiate an object of this class and specify the in its Listener.

\section{IMPLEMENTATION}

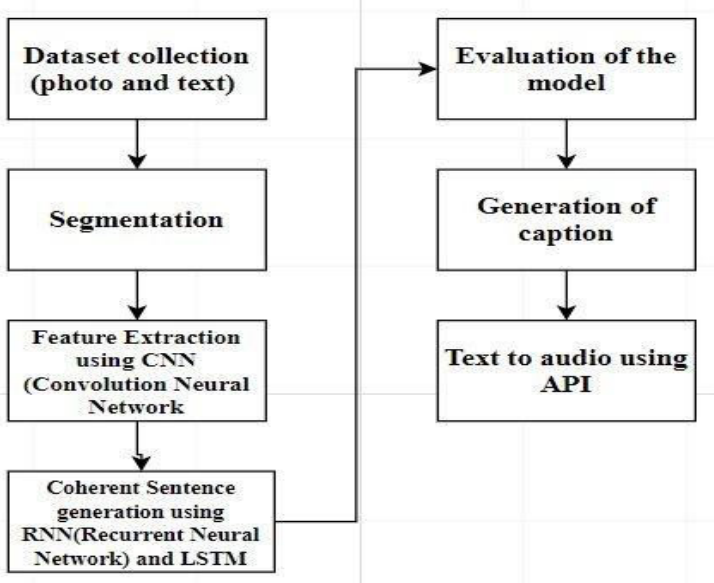

Fig 4. Block Diagram of the System
The very first step of implementation is importing all the necessary packages. The implementation of the project as shown in Fig. 4 is as follows.

1.Getting and performing data cleaning:

The Flickr8k text dataset is used for the purpose of training. The document is loaded and the contents inside the file are read into a string. A description dictionary is created that maps the images in the Flickr8k dataset with a dataset of captions. Then data cleaning is performed on all the descriptions which involve removing punctuation, converting all text to lowercase and removing words that contain numbers. After this, all the unique words are separated and the vocabulary is created from all the descriptions. Finally, a preprocessed list of descriptions is created which is stored in a file.

\section{Extracting the feature vector from all images:}

This method is often called transfer learning in which pretrained model which has already been trained on large datasets is used and features are derived from it. We use the VGG16 imaging model for the same thing.

3.Lading dataset for Training the model:

This module contains various functions which:

a. Load a text file containing a list of6000imagenames that are used for string training and return a list of image names.

b. Build a dictionary with the captions for each photo from the list of images. For each caption, the<start> and <end> Identifiers are appended. This is required for the LSTM model to define the start and end of the caption.

c.Gives the dictionary for image names and their feature vectors which were previously extracted. The max_length is calculated to decide the model structure parameters. Max_length of description is 34 .

4.Tokenizing the vocabulary and creating data generator: Each word of the vocabulary with a unique index value and saved to a pickle file. The model is trained on 6000 images. The sum of datafor6000imagescannot be stored in memory such that a generator process is used to produce batches.

TABLE 1.

EXAMPLE OF INPUT AND OUTPUT TO THE MODEL

\begin{tabular}{|c|c|c|}
\hline X1 (feature vector) & X2(text sequence) & $\begin{array}{c}Y \text { (word to } \\
\text { predict) }\end{array}$ \\
\hline feature & start, & two \\
\hline feature & start, two & dogs \\
\hline feature & start, two, dogs & drink \\
\hline feature & start, two, dogs, drink & water \\
\hline feature & start, two, dogs, drink, water & end \\
\hline
\end{tabular}


Table 1 shows an example in which the input to the model is [x1, x2] and the output will be $\mathrm{y}$, where $\mathrm{x} 1$ is the 2048 feature vector of that image, $\mathrm{x} 2$ is the input text sequence and $\mathrm{y}$ is the output text sequence that the model has to predict.

\section{Defining the CNN-RNN model:}

The structure of the model consists of three major parts:

1.Feature Extractor - The attribute obtained from the image has a size of 2048 , with a thick layer, which restricts the dimensions to 256 nodes.

2.Sequence Processor - The embedding layer manages the text input, followed by the LSTM layer.

3.Decoder -By combining the output from the two layers above, we're going to process the thick layer to create the final estimate. The final layer would have the same number of nodes as the scale of the vocabulary.

\section{Training the model and evaluation:}

To train the model, 6000 training images have been used by generating the input and output sequences in batches and fitting them to the model using model.fit_generator () method. The BLEU metric is used for evaluating and testing the performance of the caption generator.

\section{CONCLUSION}

The proposed system generates captions for photos taken on a smartphone. The system is specifically built for helping blind people in knowing what is happening in their surroundings. The system operates by sending an image to a server where all the processing is done by using deep learning algorithms like CNN, RNN, and LSTM. The blind people will be benefited from this app as the app will describe the surroundings in the form of audio. Improvisations would be made in order to expand the recognition ability of the system. These include the ability of the conversion of continuous captions into the video and many more. Another feature that could be added is to learn more from the feedback provided by the user after using the application.

\section{REFERENCES}

[1] Feng, Y., \& Lapata, M. (2013). Automatic Caption Generation for News Images. IEEE Transactions on Pattern Analysis and Machine Intelligence, 35(4), 797812.

[2] Vijay, K., \& Ramya, D. (2015). Generation of caption selection for news images using stemming algorithm. 2015 International Conference on Computation of Power, Energy, Information and Communication
(ICCPEIC).

[3] Ramnath, K., Baker, S., Vanderwende, L., El-Saban, M., Sinha, S. N., Kannan, A., Torresani, L. (2014). AutoCaption: Automatic caption generation for personal photos. IEEE Winter Conference on Applications of Computer Vision.

[4] Karpathy, A., \& Fei-Fei, L. (2017). Deep VisualSemantic Alignments for Generating

Image Descriptions. IEEE Transactions on Pattern Analysis and Machine Intelligence, 39(4)664-676.

[5] Vinyals, O., Toshev, A., Bengio, S., \& Erhan, D. (2015). Show and tell: A neural image caption generator. 2015 IEEE Conference on Computer Vision and Pattern Recognition (CVPR).

[6] Drossos, K., Adavanne, S., \& Virtanen, T. (2017). Automated audio captioning with recurrent neural networks. 2017 IEEE Workshop on Applications of Signal Processing to Audio and Acoustics.

[7] Poghosyan, A., \& Sarukhanyan, H. (2017). Shortterm memory with read-only unit in neural image caption generator. 2017 Computer Science and Information Technologies (CSIT).

[8] Vishwash Batra, Yulan He, Neural Caption Generation for News Images (2018) George Vogiatzis School of Engineering and Applied Science, Aston University

[9] Galvez, R. L., Bandala, A. A., Dadios, E. P., Vicerra, R. R. P., \& Maningo, J. M. Z. (2018). Object Detection Using Convolutional Neural Networks. TENCON 2018 - 2018 IEEE Region 10 Conference.

[10] Xu, N., Liu, A.-A., Wong, Y., Zhang, Y., Nie, Y., \& Kankanhalli, M. (2018). Dual-Stream Recurrent Neural Network for Video Captioning. IEEE Transactions on Circuits and Systems for Video Technology, 1-1.

[11] Saha Sumit, (2015, Dec). A Comprehensive Guide toConvolutional Neural NetworkstheELI5 way.towardsdatascience.Retrievedfromhttps://towardsdata science.com/ 